\title{
Caracterización de geotextiles usando Microscopia Electrónica de Barrido
}

\section{Characterization of geotextiles with Scanning Electron Microscope}

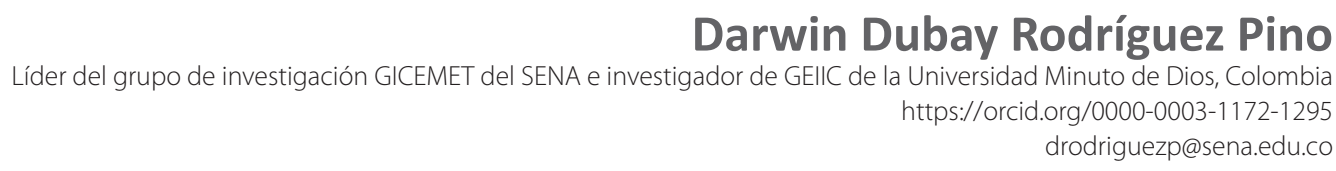

Henry Yecid Bustos Castañeda Docente e investigador del grupo de investigación GEIIC de la Universidad Minuto de Dios, Colombia https://orcid.org/0000-0003-2983-9910 hbustos@uniminuto.edu

Martin Eduardo Espitia Nery

Docente y líder del grupo de investigación GEIIC de la Universidad Minuto de Dios, Colombia https://orcid.org/0000-0001-5626-3051

mespitia@uniminuto.edu

Fecha de recepción: 12 de junio del 2018

Fecha de aceptación: 17 de septiembre de 2018

Sugerencia de citacion: Rodríguez Pino, D., Bustos Castañeda-H. y Espitia Nery, M. (2018).

Caracterización de geotextiles usando microscopia electrónica de barrido. Mutis, 8(1) 7-16, doi: http://dx.doi.org/10.21789/22561498.1372

Editor: Javier Hernández Fernández

\section{RESUMEN}

Los geotextiles son materiales sintéticos de gran deformabilidad compuestos por fibras poliméricas. Estos se asemejan a los textiles (telas) y pueden ser enrollados, cortados y cosidos. Son empleados para obras de ingeniería, particularmente en aplicaciones geotécnicas. Existen dos tipos de geotextiles: los tejidos y los no tejidos, lo cuales son utilizados en aplicaciones de filtración, drenaje, separación, reforzamiento, barrera y protección. Este trabajo desarrolla un proceso de caracterización usando microscopia electrónica de barrido (MEB) y espectroscopia de energía dispersa, con el fin de determinar características morfológicas y químicas de dos tipos de geotextiles: FORTEX y FIBERTEX. A partir del análisis espectrográfico se encuentra la distribución de los elementos químicos en estos materiales. Así mismo, a través de la observación con microscopia electrónica se identifican las características morfológicas que permiten establecer el potencial de los geotextiles para nuevos usos en la industria electrónica, tales como la generación de energía y la impresión de microcircuitos y dispositivos en geotextiles.

Palabras clave: geotextiles, microscopia electrónica de barrido, espectroscopia de energía dispersa, FORTEX, FIBERTEX, tejidos. 


\begin{abstract}
Geotextiles are highly deformable synthetic materials formed by polymeric fibers similar to a fabric. As their name suggests, geotextiles are similar to textiles and can be rolled, cut and sewed. These are used for engineering works, particularly in geotechnical applications. There are two types of geotextiles: tissues and nonwovens, which are used for filtration, drainage, separation, reinforcement, barrier-building and protection. This work develops a characterization using scanning electron microscopy (SEM) and energy dispersive spectroscopy (EDS) in order to identify the morphological and chemical characteristics of two types of geotextiles: FORTEX and FIBERTEX. Chemical elements distribution and morphological characterization are based on spectrographic analysis. This allows establishing the potential of geotextiles for new uses in the electronics industry, such as energy generation on roads and the printing of microcircuits and electronic devices in geotextile fibers.
\end{abstract}

Keywords: Geotextiles, scanning electron microscopy, energy disperse spectroscopy, FORTEX, FIBERTEX, fabrics.

\title{
NOMENCLATURA
}

Microscopio electrónico de barrido (MEB): scanning electron microscopy (SEM).

Espectroscopia de energía dispersa (EED): energy dispersive spectroscopy (EDS).

\section{INTRODUCCIÓN}

Desde la antigüedad, los textiles han sido usados por el hombre en múltiples aplicaciones. Durante el desarrollo de la humanidad, se observa que los usos más comunes de estos materiales han tenido fines domésticos y funerarios (Anand, 2000). La evolución de la tecnología textil y cómo las formas de los tejidos cambiaron en la edad de bronce y la edad de hierro son factores relevantes para comprender la importancia de estas materias primas. También es necesario considerar cómo las diferentes invasiones territoriales e intercambios culturales no solo permitieron la evolución de la tecnología sino además su transferencia en determinadas culturas.

La evolución de los textiles se resume en una línea del tiempo que se remonta a la prehistoria, cuando los primeros hombres usaban cuero de animal para cubrirse del frío y como protección ante las condiciones climáticas. Es solo hasta la edad de bronce que los antiguos fenicios empezaron a desarrollar técnicas de hilado de fibras naturales, con el fin de mejorar las propiedades de las cubiertas. Luego, durante la edad de hierro, se comenzaron a implementar técnicas de tejido artesanal (Bhushan, 2010).

La época más representativa de la evolución textil corresponde a aquella en que las culturas más representativas de la antigüedad (egipcia, fenicia, china, tailandesa, japonesa, bizantina, entre otras) lograron la fabricación masiva de textiles y la aplicación de técnicas de estampado y color (GEO Matrix, 2017).

El crecimiento de la industria textil ha tenido hitos importantes desde la edad industrial, ya que los desarrollos tecnológicos han permitido el mejoramiento de las técnicas de fabricación y la optimización de la producción de fibras y textiles. Para el año 2000, la producción mundial de este sector alcanzaba los $\$ 60$ billones de dólares y presentaba una expectativa de crecimiento de $3,8 \%$ (The Bombay Textile Research Association, 2012; Velde, 2008).

Adicionalmente, desde 1997 se ha presentado un auge por avanzar en el desarrollo de textiles técnicos ${ }^{1}$ (COTEC, 2014), los cuales contienen componentes tecnológicos más avanzados que permiten adicionar y transformar los textiles en materiales con propiedades especiales. Es así como los ingenieros y profesionales de diferentes áreas han realizado esfuerzos por generar avances en este tipo de materias primas, buscando satisfacer las necesidades propias de cada sector. A partir de tales esfuerzos, por

1 Textiles técnicos: textiles especializados con propiedades diferentes a los textiles regulares que entregan valor agregado a los materiales. 
ejemplo, ha sido posible fabricar geotextiles, para lo cual se requiere de un alto nivel de conocimiento en áreas como la ingeniería civil (Anand, 2000; Jahan, 2018). Así mismo, estos desarrollos han permitido la generación de textiles para la industria médica y otras industrias debido a las propiedades especiales de este tipo de fibras, entre las que destaca la resistencia al fuego (típicamente empleados por bomberos), la protección antibalas (para uso policial) y otras aplicaciones (DuPont Engineering Polymers, 2000). A diferencia de otros tipos de hilos para la obtención de textiles, los hilos de los geotextiles se clasifican entre las fibras químicas, ya que generalmente son obtenidos de manera artificial.

La investigación sobre geotextiles es escasa y la información sobre materiales específicos para su elaboración es reducida. Como muestra de ello, la búsqueda en la base de datos scopus arroja tan solo 22 resultados (scopus, 2018) para la palabra clave "geotex", mientras que las bases ScienceDirect y Springerlink arrojan 35 y 36 resultados, respectivamente. Además, al combinar la búsqueda entre "geotextil" y "SEM", no se presenta resultado alguno. Por esta razón, resulta relevante llevar a cabo exploraciones sobre estos materiales a través de SEM y EDS.

Este documento presenta la caracterización de dos tipos de geotextiles (FORTEX y FIBERTEX) usando microscopia electrónica de barrido (MEB) y espectroscopia de energía dispersa (EDS). El estudio del cual deriva este artículo permitió descubrir propiedades y características morfológicas y químicas de estas fibras que potencialmente permitirán su uso en aplicaciones no convencionales, tales como la generación de energía y la impresión de microcircuitos, con el fin de generar valor agregado en la construcción de vías y obras civiles.

\section{MATERIALES Y MÉTODOS}

A continuación, se explica la metodología experimental empleada para el desarrollo del presente estudio, teniendo en cuenta el tratamiento de las muestras, el montaje del microscopio electrónico de barrido, las condiciones experimentales de la caracterización y los parámetros técnicos configurados para la observación de las muestras. Como parte de la caracterización de los tejidos que comprenden la estructura que conforma los geotextiles, se siguen estrictamente los parámetros de temperatura y humedad ambientales comprendidos en un rango de $18-20$ ㄷ C y $40-50 \% \mathrm{HR}$.

La Figura 1 muestra el flujo de la metodología usada, señalando la secuencia desde la preparación de las muestras, pasando por el uso de técnicas de caracterización, el desmontaje de muestras y el análisis de la información.

Figura 1. Metodología y flujo de trabajo para el proceso de experimentación.

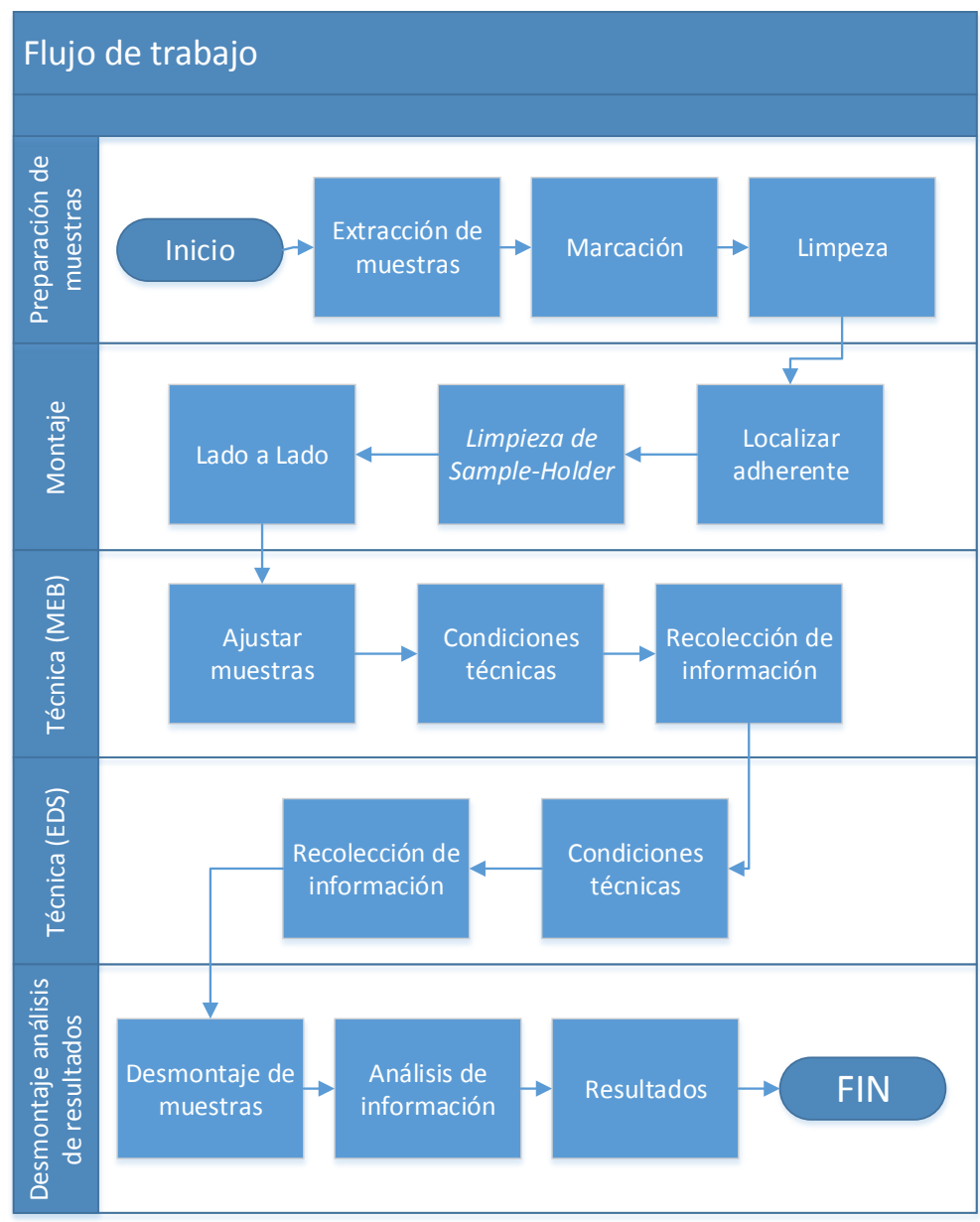

\section{Preparación de muestras}

Para la preparación de las muestras se tuvo en cuenta la estructura de las fibras de los geotextiles, con el fin de no afectar las características morfológicas y químicas durante el proceso de preparación y montaje. Las características principales de los 2 geotextiles usados son: 
Tabla 1. Características de geotextiles objeto de análisis.

\begin{tabular}{|c|l|}
\hline $\begin{array}{c}\text { Nombre } \\
\text { del } \\
\text { geotextil }\end{array}$ & \multicolumn{1}{c|}{ Características y aplicaciones } \\
\hline FIBERTEX & $\begin{array}{l}\text { Geotextil no tejido, con fibras vírgenes de polipropileno en seco, extruidas, cardadas y } \\
\text { punzonadas, altas propiedades de abrasión, alta resistencia dimensional y uniformidad, } \\
\text { resistencia a altas temperaturas, elongación superior a 50\%. Aplicaciones en filtros, } \\
\text { drenajes y separación de suelos para la construcción y protección de geo membranas. }\end{array}$ \\
\hline FORTEX & $\begin{array}{l}\text { Geotextil tejido con fibras de multifilamento de poliéster de alta tenacidad (PET), } \\
\text { caracterizado por presentar alto desempeño mecánico e hidráulico. Resistencia a U.V } \\
\text { componentes biológicos y químicos presentes en los suelos. Aplicaciones en vías, } \\
\text { aeropuertos, terraplenes ferrocarriles, edificaciones, diques, presas, parqueaderos, } \\
\text { áreas deportivas, plataformas, muros de contención, taludes de alta pendiente, canales, } \\
\text { márgenes de ríos, laderas, presas, puertos, bermas y aproches. }\end{array}$ \\
\hline
\end{tabular}

Las muestras son preparadas a partir de un corte realizado con tijeras de costura. Para ello, se siguieron los siguientes pasos:

- Extracción de dos secciones de fibra FORTEX BX30.
- Limpieza de la muestra usando nitrógeno (lado A y lado B).

- La manipulación de las muestras se realiza usando guantes de nitrilo durante todo la etapa de manipulación de muestras (Figura 2).

- Extracción de dos secciones de fibra FIBERTEX F20.

Figura 2. Resultado de muestras preparadas, montaje y manipulación.

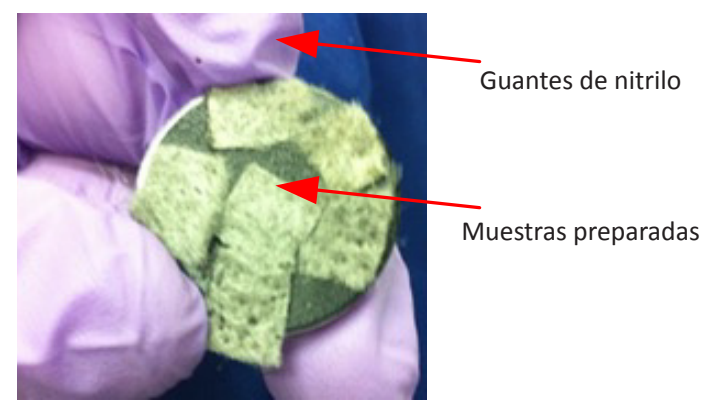

\section{Montaje}

El montaje de las muestras para realizar tanto el análisis del lado A como B de cada muestra es realizado sobre un Sample-Holder de $13 \mathrm{~mm}$ de radio, el cual puede ser empleado en microscopios electrónicos de barrido (Figura 3). Además, se usó cinta conductora de carbono para adherir las muestras de geotextil al Sample-Holder del microscopio, esto con el fin de evitar que las muestras se carguen electrostáticamente de forma instantánea. La Figura 4 ilustra dos tipos de Sample-Holders: el de la izquierda corresponde al Sample-Holder de un microscopio electrónico de barrido JEOL-6010LA, el de la derecha es un Sample-Holder Phenom XL. 
Fig ura 3. A: Sample-Holder con montaje de geotextil FIBERTEX en equipo JEOL-6010LA. B: Sample-Holder con montaje de geotextil FORTEX en equipo Phenom XL.

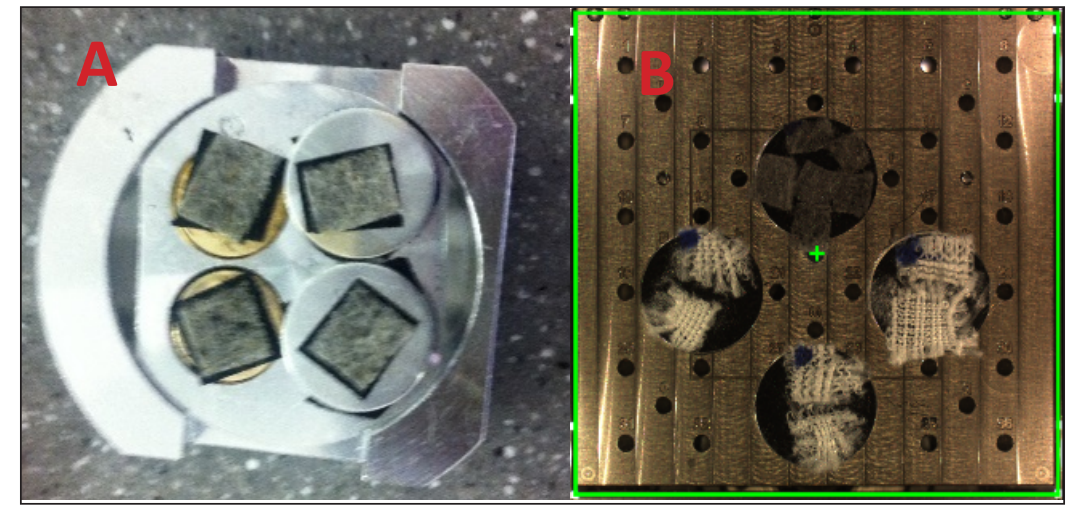

\section{Técnica de caracterización con MEB}

Para la observación de las muestras se usaron dos tipos de microscopios electrónicos de barrido. En el primer barrido se usó un equipo JEOL-6010LA. Para la segunda etapa con integración del EDS, se usó un Phenom XL.

Figura 4. A: Microscopio electrónico de barrido SEM JEOL-6010LA. B: Microscopio electrónico de Barrido SEM Phenom XL.

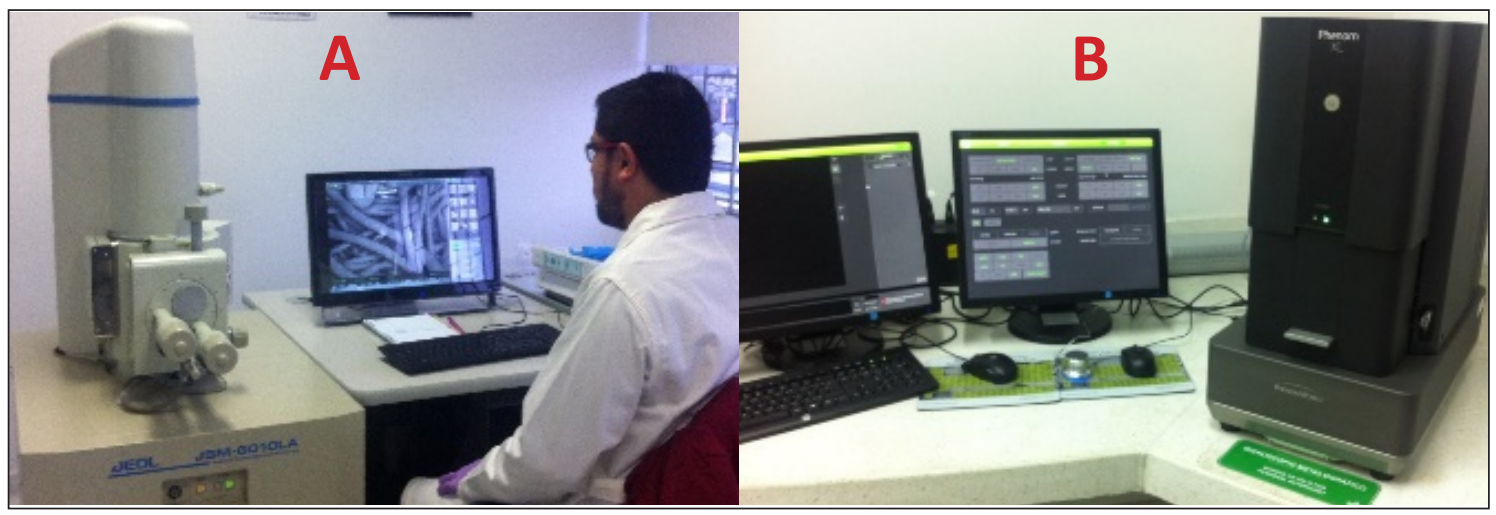

A continuación, se describe el procedimiento general efectuado:

- Se procede a generar vacío en la cámara del SEM (aplica para ambos modelos: modo bajo vacío).

- Se configuran las condiciones de rayo como voltaje (15 kV), las condiciones de cámara y de magnificación.

- Se toma imagen a 500X, 1.000X (Figura 5), 2.000X, $5.000 x$ y $10.000 x$.

- Luego de registrar la información se procede a estabilizar la presión de la cámara y posteriormente se realiza el retiro de muestras.
Con el fin de lograr las condiciones técnicas necesarias para el análisis, se selecciona un rango de magnificación que no supere $10.000 \mathrm{X}$, puesto que la intensidad del haz de electrones se concentraría en un área más reducida, generando así un deterioro del material de la muestra y una carga electrostática no deseada.

Las imágenes captadas de las fibras FORTEX muestran que estas tienen un diámetro estimado de $25 \mu \mathrm{m}$ $\pm 5 \mu \mathrm{m}$, mientras que en las fibras FIBERTEX existe mayor dispersión en las dimensiones, así como menor homogeneidad entre fibras, con un diámetro estimado de $28 \mu \mathrm{m} \pm 11 \mu \mathrm{m}$. 
Figura 5. Imagen capturada con SEM Phenom XL de geotextil FORTEX a magnificación de $1.000 X$.

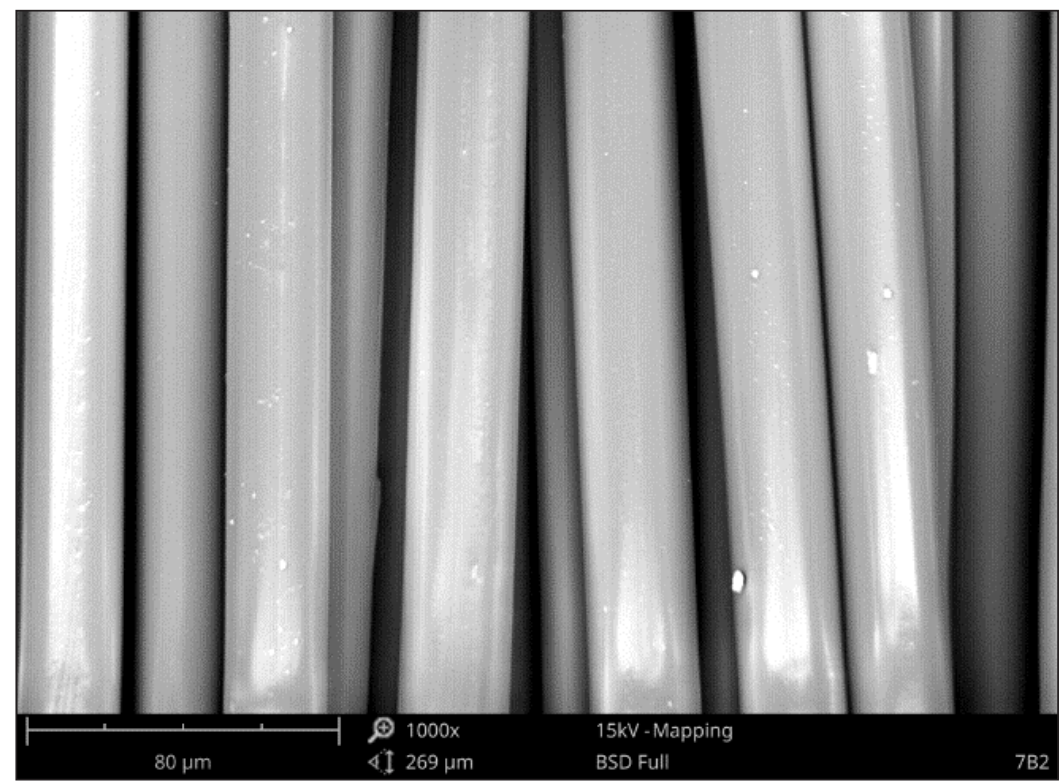

\section{Técnica de caracterización con (EDS)}

Como instrumento de análisis espectrográfico se usó el EDS del modelo Phenom XL, sistema que contiene una resolución de energía de $<=137 \mathrm{eV}$, un área activa de detección de $25 \mathrm{~mm}^{2}$ y una ventana de $\left(\mathrm{Si}_{3} \mathrm{~N}_{4}\right)$, aplicando el siguiente procedimiento:

Luego de tomar la imagen usando SEM, se procede a ejecutar un barrido o mapping ${ }^{2}$ sobre la misma muestra y bajo las mismas condiciones.

Se aplica un barrido normal que por muestreo toma alrededor de 5 minutos.

Se genera el registro de la información de mapping, espectrografía y distribución proporcional de los elementos químicos identificados (ver Figura 6).

Cada uno de los elementos identificados contiene una intensidad de energía que está relacionada con la cantidad de energía que se dispersa y que es capturada por el sensor. De esta forma se identifica que los picos más altos representados en el espectro corresponden a los materiales con alta probabilidad de localizarse en el punto analizado.

2 Mapping: rutina de barrido para análisis de espectro de energía dispersa en varios puntos de una muestra, en un recorrido bidimensional o en una superficie en dos dimensiones.
Al someter las fibras a una exposición prolongada del haz de electrones (tiempo promedio de 2 minutos) a una magnificación que oscila entre $5.000 \mathrm{X}$ y $10.000 \mathrm{X}$, se encuentra que los patrones de alineación del haz de electrones generan patrones lineales de aproximadamente $250 \mathrm{~nm}$ de ancho. En la Figura 7 se ilustra la imagen de una muestra de fibra FORTEX sometida a una intensidad del haz de electrones a 10 kV, magnificación de 5.000X y una exposición de 3

Figura 6. A: mapping de geotextil FIBERTEX. B: espectro con distribución y cantidad de energía dispersa detectada por elemento identificado. C: tabla de elementos encontrados y su distribución porcentual en la muestra.

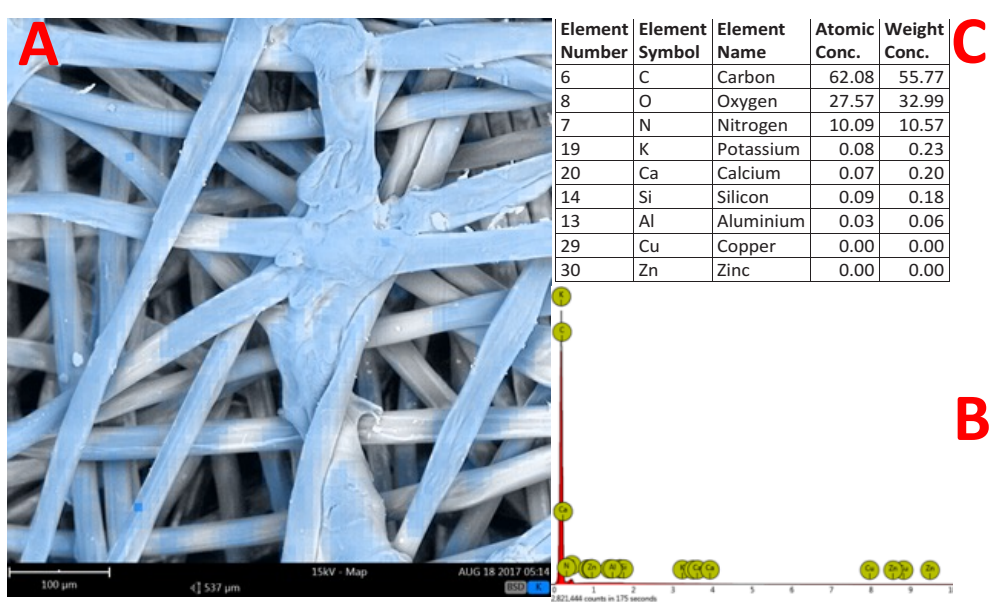


minutos. La imagen permite evidenciar la afectación lateral generada y la degradación del material de las fibras que componen este tejido. Esta reacción puede ser aprovechada para generar litografía de manera muy similar a como se realiza usando focus ion beam lithography (FIB).

Figura 7. Imagen de geotextil FORTEX sometida a intensidad prolongada del haz de electrones, magnificación de 5.000X.

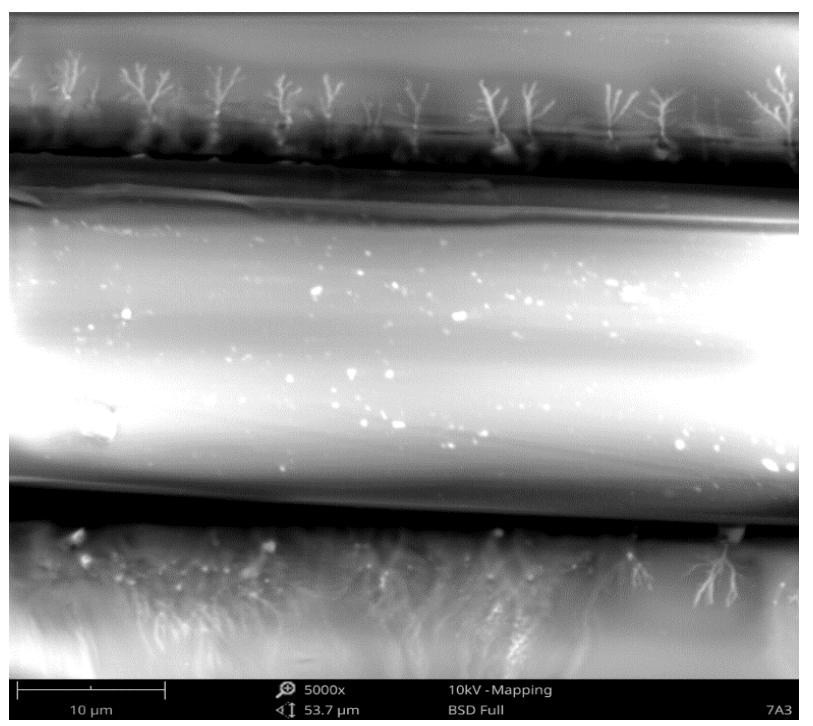

Para lograr integrar procesos de fabricación a nano escala sobre este material se requiere una caracterización del mismo que permita lograr una intervención controlada, con lo cual se pueda llevar a cabo litografía a escalas micro y nanométricas. Las posibilidades por descubrir son infinitas. Tanto es así que de lograr micro o nanolitografía sobre este material se podría realizar impresión de microcircuitos, modelamiento de micro y nanodispositivos, generación de energía con materiales piezoeléctricos, así como microsensores, microcanales, un sinnúmero de aplicaciones derivadas, y funcionalidades integradas en los geotextiles.

Aunque el alcance de este trabajo no llega a la caracterización del material para lograr micro y nanolitografía estructurada con condiciones plenamente definidas, se plantean hipótesis de las cuales derivan posibles trabajos futuros enfocados en explorar las posibilidades de las fibras y los tejidos de los geotextiles.
Existen varios trabajos en la literatura sobre micro y nanolitografía, así como sobre aplicaciones ya desarrolladas mediante el uso de técnicas tanto de caracterización como de fabricación. Una de las referencias más usadas es el cuaderno o handbook de nanotecnología de Barath Bhushan (2017), el cual muestra aplicaciones y técnicas que permiten lograr la fabricación de micro y nanodispositivos. En cuanto a la contribución de este trabajo, vale la pena mencionar que este se concentra en la determinación del potencial de los materiales empleados en los geotextiles para la generación de nuevas aplicaciones, a través de la integración de micro y nanotecnología.

Durante los procesos de caracterización de materiales con microscopia electrónica de barrido, los materiales se ven sometidos a un haz de electrones con una configuración de $10 \mathrm{kV}$ y una magnificación de los 10.000X, tal como se muestra en la Figura 8, en donde se evidencian marcas lineales que fueron detectadas a una magnificación de $15.000 \mathrm{X}$ a $10 \mathrm{kV}$ con una exposición de 1 minuto, aproximadamente, generando este patrón sobre la fibra que permite evidenciar el efecto litográfico sobre el mismo.

Figura 8. Litografía identificada en geotextil FORTEX cuadrante generado a $10.000 \mathrm{X}-10 \mathrm{kV}$. Se observan nanopartículas y trazas de litografía.

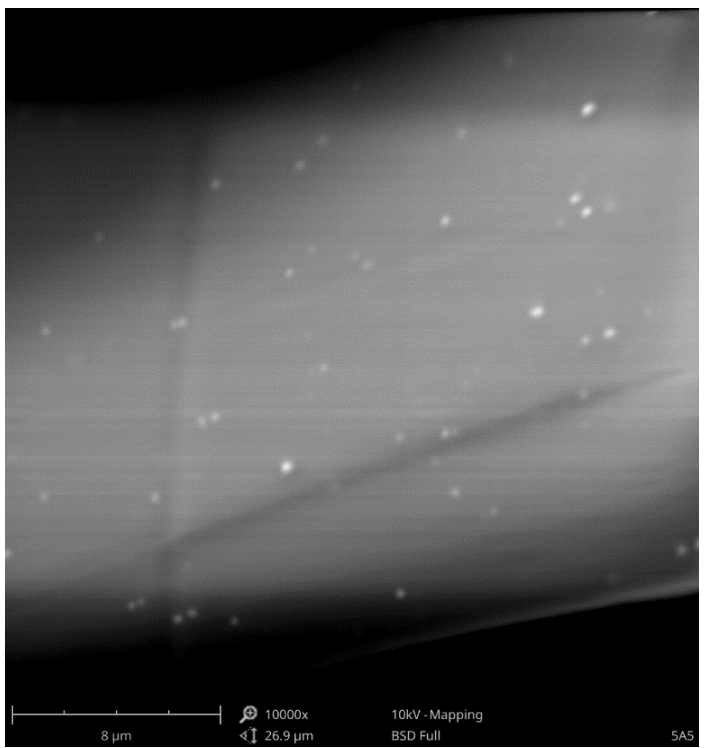

Durante el proceso de caracterización también se observaron impurezas en el material del orden de los $500 \mathrm{~nm}$ a $2 \mu \mathrm{m}$ de diámetro. Estas irregularidades se 
presentan a pesar de aplicar estrictos protocolos de limpieza, los cuales son, sin embargo, insuficientes para remover partículas de esta magnitud. Por otra parte, se encuentra mayor homogeneidad en la distribución de las impurezas para el geotextil FORTEX, en comparación con el tejido FIBERTEX. Esto permite deducir que las fibras FORTEX tienen menor adherencia de partículas que las fibras FIBERTEX. Adicionalmente, se observa que las características morfológicas en tamaño de fibras son similares. Sin embargo, la distribución y tipo de tejido son distintos, puesto que los tejidos FIBERTEX son entrelazados y no uniformes, con aditivos incorporados, mientras que las fibras FORTEX presentan entrelazado uniforme sin aditivos. Esta característica es importante a la hora de integrar micro y nanodispositivos que se adhieran a las fibras.

\section{RESULTADOS}

Con el muestreo realizado, se encontró que del $98 \%$ de los resultados del mapping aplicando EDS a los geotextiles FORTEX, más del 70\% están compuestos por oxígeno y más del $20 \%$ por carbono. En la muestra FIBERTEX, se encontró que del $97 \%$ del muestreo más del $60 \%$ es carbono, $25 \%$ oxígeno y $5 \%$ nitrógeno. Cabe resaltar que las fibras FORTEX son fabricadas en Colombia, mientras que las FIBERTEX son importadas de Alemania. En la Figura 9 se observan los dos geotextiles objeto de estudio a una misma magnificación (500X). En la Tabla 2 se muestra el resumen comparativo de ambos materiales.

Figura 9. A: imagen del geotextil FoRTEX (tejido tomado con SEM a 500X). B: imagen del geotextil FIBERTEX (no tejido, tomada con SEM a 500X).

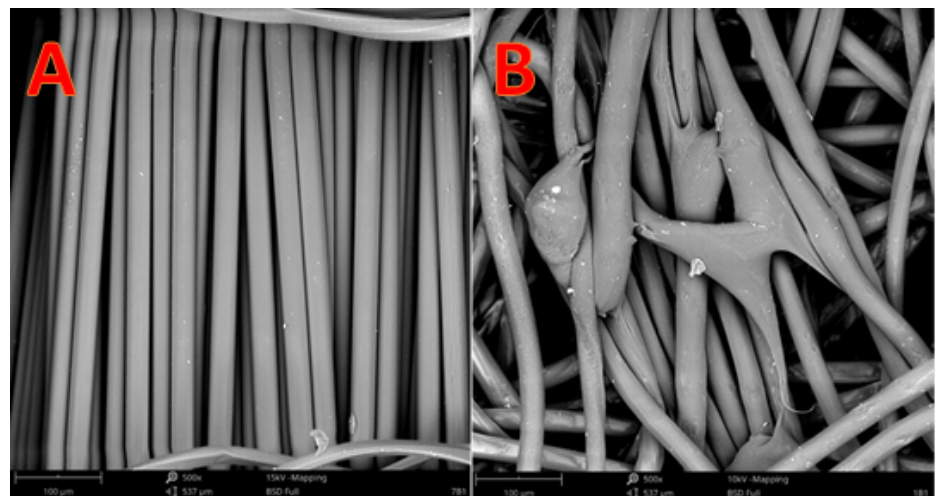

Tabla 2. Análisis de las características morfológicas y químicas de los geotextiles objeto de estudio.

\begin{tabular}{|c|c|c|c|}
\hline $\begin{array}{c}\text { Nombre del } \\
\text { geotextil }\end{array}$ & Aplicaciones & $\begin{array}{c}\text { Características } \\
\text { morfológicas }\end{array}$ & Características químicas \\
\hline $\begin{array}{c}\text { FIBERTEX } \\
\text { (imagen B) } \\
\text { Material } \\
\text { importado }\end{array}$ & $\begin{array}{c}\text { Geotextil no tejido, con fibras vírgenes de } \\
\text { polipropileno en seco, extruidas, cardadas y } \\
\text { punzonadas, altas propiedades de abrasión, alta } \\
\text { resistencia dimensional y uniformidad, resistencia } \\
\text { a altas temperaturas, elongación superior al 50\%. } \\
\text { Aplicaciones en filtros, drenajes y separación } \\
\text { de suelos en la construcción y protección de } \\
\text { geomembranas. }\end{array}$ & $\begin{array}{l}\text { Al ser un geotextil no tejido, se } \\
\text { observa una clara dispersión } \\
\text { en las fibras que conforman } \\
\text { el geotextil, así como la } \\
\text { presencia de aditivos que } \\
\text { mejoran las propiedades } \\
\text { mecánicas y químicas. }\end{array}$ & $\begin{array}{l}\text { Se encuentra que el geotextil } \\
\text { está compuesto en promedio } \\
\text { por } 60 \% \text { de carbono, } 25 \% \text { de } \\
\text { oxígeno, } 5 \% \text { de nitrógeno y } \\
\text { el restante } 10 \% \text { corresponde } \\
\text { a otros materiales como el } \\
\text { potasio, el calcio y en silicio. }\end{array}$ \\
\hline
\end{tabular}




\begin{tabular}{|c|c|c|c|}
\hline $\begin{array}{c}\text { Nombre del } \\
\text { geotextil }\end{array}$ & Aplicaciones & $\begin{array}{c}\text { Características } \\
\text { morfológicas }\end{array}$ & Características químicas \\
\hline $\begin{array}{c}\text { FORTEX } \\
\text { (imagen A) } \\
\text { Material } \\
\text { nacional }\end{array}$ & $\begin{array}{l}\text { Geotextil tejido con fibras de multifilamento de } \\
\text { poliéster de alta tenacidad (PET, caracterizado por } \\
\text { presentar alto desempeño mecánico e hidráulico. } \\
\text { Resistencia a UV componentes biológicos y } \\
\text { químicos presentes en los suelos. } \\
\text { Aplicaciones en vías, aeropuertos, terraplenes } \\
\text { ferrocarriles, edificaciones, diques, presas, } \\
\text { parqueaderos, áreas deportivas, plataformas, } \\
\text { muros de contención, taludes de alta pendiente, } \\
\text { canales, márgenes de ríos, diques, laderas, presas, } \\
\text { puertos, bermas y aproches. }\end{array}$ & $\begin{array}{l}\text { Al pertenecer a los geotextiles } \\
\text { tejidos, se observa una } \\
\text { morfología organizada de } \\
\text { las fibras que conforman } \\
\text { el geotextil, así como la no } \\
\text { presencia de aditivos. }\end{array}$ & $\begin{array}{l}\text { Se encuentra que el geotextil } \\
\text { está compuesto en promedio } \\
\text { por } 70 \% \text { de oxígeno, } 20 \% \\
\text { de carbono y el restante } \\
10 \% \text { corresponde a otros } \\
\text { materiales como el nitrógeno. }\end{array}$ \\
\hline
\end{tabular}

Se encontró que las fibras FORTEX reaccionaron a la presencia del haz de electrones de forma altamente sensible, lo cual hizo que este material respondiera y presentara características que permitieron su modificación a micro y nanoescala; propiedad que no fue identificada en las fibras FIBERTEX.

Se encontró que las fibras FORTEX reportan menor adherencia de partículas de impurezas entre $500 \mathrm{~nm}$ y $2 \mu \mathrm{m}$ de diámetro que las fibras FIBERTEX, característica que resulta importante a la hora de adherir micro o nanodispositivos a estas fibras.

\section{CONCLUSIONES}

Al encontrar características comparativas entre dos tipos de geotextiles a microescala, así como la aplicación de parámetros y procedimientos para la caracterización de este tipo de materiales poco explorados en otras aplicaciones, se encontró que las posibles aplicaciones en otras áreas se deben a las características morfológicas y químicas que estos geotextiles poseen. Por ello, características como la sensibilidad al haz de electrones posiblemente permitirán el uso de nanolitografía en este tipo de fibras.

De los resultados obtenidos, se puede inferir que las diferencias a nivel morfológico entre los dos geotextiles analizados se encuentran directamente relacionadas con el hecho de que sean tejidos o no tejidos, así como con sus propiedades físico-químicas, lo cual se ve representado en el tipo de aplicaciones determinadas por sus propiedades mecánicas y químicas, tales como una gran resistencia a altas temperaturas, permeabilidad, abrasión, desempeño mecánico e hidráulico, resistencia a rayos UV entre otros (ver Tabla 2).

\section{TRABAJOS FUTUROS}

Una de las finalidades del trabajo global es encontrar una correlación y trazabilidad entre las propiedades a macro, micro y nanoescala, así como determinar propiedades especiales a micro y nanoescala que permitan dar nuevos usos a estas fibras en otras áreas y a través de distintas aplicaciones, tales como la impresión de microcircuitos que permitan la generación de energía por cambios de presión en su superficie (principio piezo-eléctrico) o la instalación de microsensores que permitan establecer puntos críticos en muros o terraplenes, ductos o vías de tráfico pesado.

Finalmente, de este trabajo puede derivar un catálogo de geotextiles con propiedades identificadas a micro y nanoescala, que contenga el análisis del uso de instrumentación típicamente usado para la caracterización de materiales a tales escalas, como es el caso de microscopios de fuerza atómica, electrónicos de barrido, rayos X, entre otros (Gates, 2018).

\section{RECONOCIMIENTOS}

Servicio Nacional de Aprendizaje (SENA): Centro Metalmecánico, Centro de Manufactura Textil y del Cuero y Centro de Materiales y Ensayos, RegionalDistrito Capital. 
Universidad Minuto de Dios: Facultad de Ingeniería, Programa de Ingeniería Civil.

Geomatrix: Empresa dedicada a la fabricación, venta e importación de geotextiles (suministro de muestras).

\section{REFERENCIAS}

Anand, A. R. (2000). Handbook of technical textiles. Cambridge, UK: CRC Press.

Bhushan, B. (2010). Handbook of Nanotechnology. New York, NY, USA: Springer.

DuPont Engineering Polymers. (2000). Technical handbook. N. C.: Dupont.

Fundación COTEC para la Innovación Tecnológica. (2014). Textiles Técnicos. Recuperado en 2018 de: http://informecotec.es/media/N31_Textiles_Tec. pdf.

Gates, W. P., Dumadah, G., \& Bouazza, A. (2018). Micro X-ray visualisation of the interaction of geosynthetic clay liner components after partial hydration. Geotextiles and Geomembranes, 46, 739-747.

GEO Matrix. (2017, 03 01). Geo textiles. Retrieved from: https://www.geomatrix.co/.

Ingold, T. (1994). Geotextiles and Geomembranes Handbook. Elsevier Ltd.

Jahan, M. W. (2018). Using a geotextile with flocculated filter backwash water and its impact on aluminium concentrations. Geotextiles and Geomembranes, 46, 759-769.

Koerner, R. (2016). Geotextiles From Design to Applications. Woodhead Publishing

SCOPUS (2018, 09 14). SCOPUS. Retrieved from: https:// www-scopus-com.ezproxy.unal.edu.co/results/ results.

uri?numberOfFields $=0 \& s r c=s \&$ clickedLink $=$ \&edit $=$ \&editSaveSearch=\&origin=searchbasic\&authorTab= \&affiliation $T a b=\& a d v a n c e d T a b=\&$ scint $=1 \&$ menu $=$ search\&tablin=\&searchterm1=geotex\&field1= TITLE_ABS_KEY\&dateTyp
The Bombay Textile Research Association. (2012). Hand Book of Geotextiles. Bombay: BTRA.

Velde, J. K. (2008). The role of textile and clothing industries in growth and development strategies. N. C.: Odi. 